\title{
L'HOMMe L'Homme
}

Revue française d'anthropologie

203-204 | 2012

Anthropologie début de siècle

\section{L'historien au pays des merveilles?}

Histoire et anthropologie au début du XXI ${ }^{\mathrm{e}}$ siècle

Historians in Wonderland? History and Anthropology at the Start of the $21^{\text {st }}$

Century

Étienne Anheim

\section{OpenEdition}

Journals

Édition électronique

URL : http://journals.openedition.org/lhomme/23239

DOI : 10.4000//homme.23239

ISSN : $1953-8103$

Éditeur

Éditions de l'EHESS

Édition imprimée

Date de publication : 4 décembre 2012

Pagination : $399-427$

ISSN : 0439-4216

Référence électronique

Étienne Anheim, «L'historien au pays des merveilles ? », L'Homme [En ligne], 203-204 | 2012, mis en

ligne le 03 décembre 2014, consulté le 03 mai 2019. URL : http://journals.openedition.org/

Ihomme/23239; DOI : 10.4000/lhomme.23239

(c) École des hautes études en sciences sociales 


\title{
L'historien au pays des merveilles? Histoire et anthropologie au début du $\mathrm{XXI}^{\mathrm{e}}$ siècle
}

\begin{abstract}
Étienne Anheim
Il est inutile d'insister, dit Alice en regardant la maison comme si elle discutait avec elle. Je refuse de rentrer. Je sais que je serais obligée de repasser à travers le Miroir... de revenir dans le salon... et ce serait la fin de mes aventures! Lewis Carroll, De l'autre côté du miroir (1871).
\end{abstract}

\section{P}

OURQUOI les historiens lisent-ils les anthropologues? La réponse a longtemps semblé évidente car la force du modèle anthropologique structural a été telle, en particulier en France, qu'elle a engendré une orientation disciplinaire devenue, sous le nom $\mathrm{d}^{\prime}$ ' anthropologie historique ", l'un des programmes de recherche dominants dans l'historiographie depuis les années 1960-1970 (Burguière 1988 [1978], 1995) ${ }^{1}$. Les historiens français ont traversé le miroir pour découvrir un champ d'investigation anthropologique dont la fécondité tenait à la différence disciplinaire et à l'ambition épistémologique et nomologique, ainsi qu'à l'espoir de constituer une discipline universitaire d'avenir. Au même moment, en Italie ou en Angleterre, d'autres historiens sont allés à la rencontre d'une anthropologie qui était sociale, comme celle de l'école de Manchester, mais aussi et surtout culturelle, comme en témoignent les cultural studies qui en sont issues ${ }^{2}$, et se sont livrés à des expérimentations

1. Pour ce qui concerne l'histoire médiévale, on trouvera un état complet de la question dans le colloque en forme de bilan, Faire l'anthropologie historique du Moyen Âge, sous la direction d'Elisa Brilli, Blaise Dufal \& Pierre-Olivier Dittmar (2010) - en particulier, du point de vue de l'historiographie de l'anthropologie historique, outre l'introduction et la conclusion, les contributions de Jean-Claude Schmitt (2010a) et Jean-Pierre Albert (2010).

2. L'influence de l'école de Manchester sur la production historique et sa filiation de long terme avec l'historicisme anthropologique contemporain mais aussi certaines formes d'histoire sociale (Cefaï, ed. 2010 ; Gribaudi 1998) sont à mettre en parallèle avec l'essor du Centre for Contemporary Cultural Studies de Birmingham à partir de 1964, autour de Raymond Williams, Richard Hoggart, Stuart Hall, ou encore des recherches d'Edward P. Thompson (1963). En Italie, le contexte .......

Je remercie, pour leur aide, leurs indications bibliographiques et leurs conseils, Patrick Boucheron, Pierre Chastang, Paul Costey, Didier Lett, Antoine Lilti, Michel Naepels et Valérie Theis. 
historiographiques tout aussi novatrices, dont le "tournant critique » des Annales s'est fait l'écho à la fin des années 1980, apportant une inflexion à l'approche héritée de l'anthropologie historique structuraliste tout en préservant l'alliance interdisciplinaire ${ }^{3}$. Ainsi, durant trois ou quatre décennies, suivre la recherche en anthropologie a coulé de source pour les historiens et de nombreux objets de recherche sont devenus communs; cette pratique de l'interdisciplinarité a permis des transferts rapides de concepts et, dans une certaine mesure, de modèles.

Sans qu'une rupture nette soit visible, la référence anthropologique a tendu ensuite à s'effacer dans le contexte des difficultés institutionnelles propres à la discipline, mais aussi de la "crise de l'histoire». Elle a été remplacée progressivement par des questions épistémologiques liées à l'échelle de l'enquête, à la généralisation, aux rapports entre histoire et mémoire, ou par des échanges avec d'autres disciplines, à commencer par la sociologie, même si cela n'a pas empêché la poursuite féconde $\mathrm{du}$ dialogue thématique anthropologico-historique ${ }^{4}$. Paradoxalement, ce relatif reflux de l'anthropologie s'est fait alors même que les travaux

[Suite de la note 2] intellectuel de naissance de la microstoria est fortement influencé par des problématiques anthropologiques, comme le montrent très bien les travaux de Carlo Ginzburg et leurs prolongements jusqu'à aujourd'hui, ou encore le fait que Le Saint lévrier de Jean-Claude Schmitt (2004 [1979]) soit traduit dans une collection de microstoria, tout en incluant une interrogation fondamentale sur la nature des liens sociaux, davantage illustrée par les œuvres de Giovanni Levi ou Maurizio Gribaudi. Ce développement parallèle explique la jonction entre problématiques sociales et culturelles au sein d'une approche anthropologico-historique, qui n'a pas eu d'équivalent en France dans un premier temps, dans la mesure où la réception des œuvres d'anthropologues comme Roger Bastide et ou Georges Balandier a été très limitée chez les historiens français, tournés principalement vers les travaux de Claude Lévi-Strauss - et dont témoigne encore l'intérêt limité porté par les historiens aux travaux comme ceux de Denys Cuche ou Erwan Dianteill, qui poursuivent aujourd'hui cette direction de recherche.

3. C'est finalement par le détour de l'Italie, de l'Angleterre ou des États-Unis que la question s'est posée aux historiens, par exemple à travers la lecture de travaux comme ceux de Carlo Ginzburg et Giovanni Levi, Richard Hoggart ou E.-P. Thompson, ou comme ceux de Clifford Geertz et Marshall Sahlins. Les propositions du " tournant critique » des Annales, ainsi que la problématique du jeu d'échelles et leur environnement interactionniste portent la marque de cette inflexion dans les liens entre anthropologie, histoire et construction sociale; à cet égard, on peut noter la coïncidence chronologique entre la traduction de La Formation de la classe ouvrière anglaise, d'E. P. Thompson (Paris, Le Seuil, 1988) et celle du Pouvoir au village, de Giovanni Levi (1989), deux auteurs mobilisés par les historiens français dans les débats des années 1990.

4. Pour un panorama d'ensemble sur les évolutions historiographiques des années 1990-2000, cf. Delacroix, Dosse \& Garcia (2007) et Delacroix et al., eds (2010), où l'on peut clairement mesurer le recul des problématiques anthropologiques classiques au sein du travail des historiens. On pourra voir aussi le colloque déjà cité (Brilli, Dufal \& Dittmar, eds 2010), ainsi que la table ronde publiée par la Revue d'histoire moderne et contemporaine (Minard et al. 2002), avec la participation de Jocelyne Dakhlia, Serge Gruzinski, Jean-Clément Martin, Michel Naepels et Michel Nassiet. Cette rencontre témoigne, entre autres choses, du statut particulier occupé par la parenté dans les liens entre anthropologie et histoire: c'est sans doute dans ce domaine que l'apport de l'anthropologie d'inspiration structuraliste, qu'il s'agisse de Claude Lévi-Strauss, de Françoise Héritier, de Françoise Zonabend ou, sur un registre critique mais inscrit dans le même paradigme, .../... 
des anthropologues marquaient une attention nouvelle à l'historicité mais aussi aux méthodes des historiens et aux documents d'archives - comme si la traversée du miroir s'effectuait cette fois dans l'autre sens et que se développait une anthropologie historiciste, confrontée aux mêmes enjeux que les historiens dans les années 1990-2000, mais sans que ces derniers s'y intéressassent autant qu'à l'anthropologie structurale ou culturelle qui l'avait précédée 5 .

Il se pourrait que les historiens aient été inconsciemment attristés de cette "fin de l'exotisme" anthropologique: à la forte différenciation dans les pratiques scientifiques, qui caractérisait les historiens et les anthropologues depuis l'après-guerre, succédait un rapprochement méthodologique et épistémologique qui pouvait paraître décevant aux yeux de ceux pour qui l'intérêt de l'interdisciplinarité venait de la différence de potentiel entre les pôles mis en contact. Dès lors que l'anthropologie s'engageait dans une approche pragmatiste et qu'elle sollicitait les archives, que pouvait-elle encore apporter de spécifique à l'historien? Cette illusion d'optique, qui explique peut-être le recul de l'intérêt pour les anthropologues chez les historiens, relève d'une conception étroitement utilitariste de l'interdisciplinarité, qui consisterait en une importation directe d'objets, de concepts et de modèles étrangers à l'histoire afin de produire des résultats scientifiques novateurs.

[Suite de la note 4] de Maurice Godelier, a été le plus durable (Burguière et al. 1986), comme le montrent, en histoire du Moyen Âge, les recherches de Régine Le Jan ou d'Anita GuerreauJalabert, ou en histoire moderne, celles de Gérard Delille, Michel Nassiet ou, plus récemment, d'Elie Haddad. Les dynamiques proprement anthropologiques de renouvellement de ce champ, qui apparaissent clairement par exemple dans le travail de Klaus Hamberger et l'introduction de la dimension spatiale dans l'approche structurale, expliquent sans doute la persistance de cette approche dans l'étude de ce domaine, qui a cependant également intégré progressivement une méthodologie plus pragmatique, à l'image du numéro spécial de la revue Médiévales coordonné par Didier Lett (2008) qu'on peut rapprocher de l'orientation méthodologique du travail de Florence Weber, dans le domaine ethnographique. On peut enfin également songer à l'héritage majeur de l'anthropologie dans le domaine de l'histoire ancienne, dans une perspective plus politique, depuis les réflexions fondatrices de Jean-Pierre Vernant ou de Pierre Vidal-Naquet jusqu'aux travaux de Pauline Schmitt-Pantel, François de Polignac ou Vincent Azoulay.

5. On pense en particulier à l'influence d'œuvres comme celle de Nicholas Thomas (1998) et celle de Johannes Fabian (2006). Dans l'anthropologie française, on pense également aux travaux de Jean Bazin (2006), Alban Bensa (2006), Michel Naepels (1998) - sans compter les réflexions de Marc Augé (1979) et les recherches longues, minutieuses et importantes de Michel Izard (2003), d'Alfred Adler (1982) ou d'Emmanuel Terray (1995) sur des royaumes africains précoloniaux -, ainsi qu'au numéro de la revue Critique dirigé par Benoît de L'Estoile et Michel Naepels(2004), mais aussi aux recherches d'orientation un peu différente, comme celles de Christian Geffray (1991, 1997 et 2001), où l'historicité tient une place tout à fait centrale, sans contradiction avec le recours aux outils analytiques (principalement lacaniens), ou de Julien Bonhomme (2005, 2009), qui ont pour point commun de donner à l'historicité une place majeure. Pour un état récent de la question du point de vue de l'anthropologie, cf. le dossier des Annales, «L'anthropologie face au temps» (Naepels, ed. 2010) et, pour un regard d'historien, Étienne Anheim (2010). 
Une grande partie de la recherche en anthropologie dans les deux dernières décennies se prête moins à cette manière de faire: quel est le gain - intellectuel mais aussi stratégique, bien sûr - à importer des notions et des concepts si voisins de ceux de l'historien? Pour autant, de nombreux historiens continuent à lire de l'anthropologie, non pas seulement par habitude ou tradition. À partir d'une expérience localisée, celle d'un historien qui s'intéresse à l'histoire sociale des formes culturelles à la fin du Moyen Âge, je voudrais essayer de montrer que rapporter chez eux des objets exotiques n'est pas la seule motivation des historiens pour aller visiter leurs voisins anthropologues.

\section{Statut et construction de la documentation}

À partir du début des années 1990, ce qu'on pourrait appeler la "question documentaire», c'est-à-dire le problème du statut et de l'élaboration des données empiriques disponibles pour l'enquête scientifique, a progressivement pris une place importante dans la réflexion des anthropologues - bien qu'elle se fût déjà manifestée au tout début des années 1930, au moment de l'institutionnalisation de la discipline (cf. Hollier 1991) -, tandis qu'au même moment, un phénomène similaire s'observait chez les historiens. La signification de ce déplacement est cependant profondément ambivalente. D'un côté, le discours sur le "retour» aux archives ou au terrain a correspondu à un repli sur l'«identité du métier », après les échanges interdisciplinaires et les audaces théoriques des trente années précédentes. Une vision pessimiste des sciences sociales dans les années 1990 pourrait voir dans ces "retours" la marque d'une double régression, vers une conception restreinte de l'identité disciplinaire d'une part, et vers un empirisme débarrassé de questions théoriques auxquelles on attribuait la responsabilité de la crise, de l'autre. Cette vision correspond en partie à une réalité, mais de l'autre côté, ce moment de recentrage sur les opérations concrètes de collecte et de traitement des données a été l'occasion de réflexions innovantes sur le statut de la documentation dans chacune des disciplines. En histoire, le statut des vestiges du passé, plus communément appelés les "sources » - les archives, mais aussi les autres ressources mobilisées par les chercheurs (enquêtes orales, documentation archéologique ou iconographique, etc.) s'est dégagé d'une conception naturaliste qui souvent prévalait jusque-là. Au lieu d'être considéré comme le réceptacle passif d'informations sur ce qui lui était extérieur, le document est devenu en lui-même le premier objet de la recherche, dans le cadre d'une réflexion sur sa matérialité, son élaboration, ses usages et sa transmission, renouvelant le problème 
épistémologique du statut des traces du passé au sein du processus de construction du savoir, dans la lignée des réflexions de Michel de Certeau (1975), Carlo Ginzburg (1989) ou Arlette Farge (1989) ${ }^{6}$.

Dès lors, le mouvement parallèle qui s'est opéré en anthropologie à propos du "terrain " prend un intérêt particulier aux yeux de l'historien. C'est d'abord sur le mode de l'analogie que la confrontation peut être conduite, comme dans le cas de l'approfondissement de la réflexion anthropologique sur le statut de l'informateur. Il ne s'agit pas seulement de développer les méthodes de la critique pour les adapter à la pratique de l'enquête orale; c'est bien plus radicalement la place et le statut des éléments de discours recueillis de cette façon, et leur hiérarchisation dans l'orientation du savoir anthropologique et son écriture, qui sont au cour du travail scientifique désormais.

L'anthropologie pragmatique montre ainsi un double niveau d'appréhension de la parole de l'enquêté : d'une part, il s'agit de considérer méthodologiquement à égalité l'ensemble des témoignages dans un contexte social, en considérant que le travail anthropologique ne consiste pas à décider de la vérité d'une situation, mais à étudier la complexité et les contradictions des récits et des relations sociales qu'ils tissent; mais d'autre part, il s'agit en même temps, souvent implicitement, d'en distinguer certains, non pas parce qu'ils sont plus vrais, mais parce qu'ils sont plus ouverts, c'est-à-dire qu'ils constituent une meilleure introduction à la situation sociale étudiée par l'anthropologue. Ce problème trouve un écho particulier dans le travail de l'historien du Moyen Âge, malgré l'écart entre sa situation de lecteur et la position d'interlocuteur de l'anthropologue. Comme l'historien de l'Antiquité, et sans doute plus systématiquement que l'historien des époques moderne ou contemporaine, l'historien médiéviste se trouve aux prises, au sein de sa documentation écrite, à des sources "narratives", comme des chroniques ou des récits, qui orientent son travail d'analyse.

Face à ces sources, la tentation est souvent grande de se limiter à une méthode critique traditionnelle, philologique et combinatoire, pour hiérarchiser la sincérité des témoignages et en tirer un récit uniforme organisant les faits réunis, une fois les textes passés au crible. Cette approche historienne n'a pas attendu les échanges avec l'anthropologie pour être critiquée, comme en témoigne le célèbre livre d'Arsenio Frugoni (1993) sur Arnaud de Brescia publié en Italie en 19547, qui précisément

6. Pour une perspective sur ce déplacement historiographique, cf. : Morsel (2003, 2007, 2009); Anheim \& Poncet, eds (2004); Castelli Gattinara \& Anheim, eds (2007).

7. Cf. l'importante introduction d'Alain Boureau, qui est aussi le traducteur, et qui met en évidence la rupture méthodologique opérée par Frugoni dans son traitement de la documentation. 
refuse à la fois la hiérarchisation des témoignages narratifs et la critique positiviste, pour tenter au contraire un portrait polyphonique accordant une égale attention à chaque témoignage. La leçon mérite toujours d'être méditée, tant elle semble rapprocher l'historien de l'anthropologue autour de la question du statut de l'information qu'ils rassemblent, malgré la différence fondamentale liée à la situation d'interlocution du second.

De plus, il importe de faire place à une autre manière de traiter ce problème, par une hiérarchisation non plus factuelle et positive, mais qu'on pourrait dire "sociologique", au sens où chaque individu donné, s'il a un égal intérêt intellectuel aux yeux du chercheur, n’a pas forcément une égale compétence et une égale efficacité de description sociale. Dans une perspective radicalement pragmatiste, soucieuse d'une description relative des points de vue, cette manière de hiérarchiser non pas les informations mais les informateurs n'est pas toujours assumée. Reste que la lecture attentive des recherches des anthropologues, ainsi que de leurs "remerciements ", souvent instructifs à cet égard, montre que certains enquêtés jouent un rôle stratégique à la fois dans l'introduction concrète du chercheur au sein de la communauté, dans son apprentissage linguistique et dans son initiation à la compréhension des règles sociales. Ce sont des " passeurs ", qui figurent souvent en tant que tels dans les livres d'anthropologues, car la question des liens avec ces personnes de chair et d'os est très présente à l'esprit des chercheurs.

Souvent moins visibles, ces "passeurs " existent aussi chez les historiens, qui n'en sont pas toujours conscients ou qui considèrent simplement que tel chroniqueur est privilégié parce qu'il est le plus complet ou le plus fiable - alors qu'en réalité, c'est souvent qu'il entretient également avec le chercheur et avec son propre contexte sociohistorique des relations de complicité et de connaissance qui le singularisent. Ainsi, la figure de Joinville domine-t-elle la biographie de Jacques Le Goff (1996) consacrée à Louis IX et les formulations de l'historien font de ce témoin si particulier un quasi-interlocuteur ${ }^{8}$, comme en témoigne un entretien accordé à Laurence Moulinier et Odile Redon (1998 : 94-95) :

8. Outre le chapitre central, au sens propre comme au sens figuré, «Le "vrai” Louis IX de Joinville», (Le Goff 1996 : 473-498), on pourra relever les nombreuses formules sur Joinville, souvent qualifié de "témoin exceptionnel ", dans l'introduction et la conclusion, par exemple pp. 16, 25 et pp. 888889, où Jacques Le Goff écrit : "Ainsi m’est venu peu à peu le sentiment - peut-être illusoire que je connaissais de mieux en mieux Louis, que je le voyais, que je l'entendais, que je devenais, en gardant de la distance, dans l'ombre, un nouveau Robert de Sorbon, un autre Joinville [...]. Je ne l'ai pas vu en rêve, mais je crois que j'aurais, comme Joinville, pu le faire». Sur ce livre, cf. le numéro Hommes de pouvoir. Individu et politique au temps de Saint Louis de la revue Médiévales (1998, 34), en particulier Patrick Boucheron (1998). 
"Q. - En vous lisant, on voit non pas un dialogue avec Saint Louis mais un échange à trois voix: Jacques Le Goff, Saint Louis, Joinville. Vous pourriez peut-être nous parler de vos rapports avec Joinville?

R. - Joinville m’a absolument subjugué. Ma première réaction en le lisant attentivement a été d'être ébloui par tout ce qu'il m’apportait sur Saint Louis. C'était ma meilleure source à l'évidence. Ensuite j'ai été frappé par la nature et la qualité du personnage ».

Georges Duby (1996), de la même manière, a érigé quelques auteurs médiévaux en informateurs privilégiés, auxquels il emprunte non seulement des illustrations, mais surtout une vision de la société qu'il réinvestit ensuite dans son propre savoir d'historien. Guillaume le Maréchal est un livre entièrement construit sur un seul récit, l'Histoire de Jean le Trouvère ; Le Dimanche de Bouvines donne une place particulière à la narration de Guillaume le Breton (Ibid.: 827-1050); bien des articles majeurs sont nourris de la fréquentation de Lambert d'Ardres et de son Historia Comitum Ghisnensium, auxquels il emprunte souvent non pas des événements, mais, plus important, le regard porté sur le monde féodal, comme il le revendique dans ses mémoires:

"J'avais demandé jusqu'alors aux documents qu’ils m’enseignent la vérité des faits dont ils avaient mission de conserver le souvenir. Il m’était vite apparu que cette vérité était inaccessible et que l'historien n'a de chance de s'en approcher qu'au niveau intermédiaire, au niveau du témoin, en s'interrogeant non point sur les faits qu'il relate, mais sur la manière dont il les a rapportés. Voici pourquoi je prête maintenant plus d'attention aux récits, aussi fantasmagoriques soient-ils, qu'aux notations "objectives", décharnées, que l'on peut glaner dans les archives. Ces récits m’apprennent davantage, et d'abord sur leur auteur, par ses louvoiements, ce qu'il peine à dire, ce qu'il ne dit pas, qu'il oublie ou qu'il cache. Or c'est à lui que je m'attache, retenant en premier lieu de ses paroles ce qu'elles révèlent de sa propre culture, de ses espérances, de ses craintes, de la façon dont il pense le monde, dont il se pense lui-même. L'image qu'il se fait de lui, voilà ce que je cherche à reconstituer [...]. Depuis que j'ai pour L'An mil rassemblé et analysé quelques-unes de ces sources que nous disons narratives, je moissonne abondamment parmi les phrases alambiquées d'un Raoul le Glabre, d'un Guibert de Nogent, d'un Lambert d'Ardres, ces écrivains superbes et bavards que méprisaient tant les positivistes parce qu'ils mentent parfois et qu'ils se trompent souvent, à propos d'une date ou d'un lieu» (Duby 1991 : 137-138).

Le rapport entretenu par l'historien avec sa documentation excède largement le cadre de l'étude critique d'un simple témoignage historique : chez beaucoup d'historiens du Moyen Âge, un certain nombre de sources narratives semble occuper une position centrale qui n'est pas seulement d'objet de recherche, mais surtout celle d'un guide dans l'interprétation d'une époque éloignée. Néanmoins, cette position est souvent passée sous silence et, d'ailleurs, des auteurs nourris d'anthropologie comme Jacques Le Goff ou Georges Duby ne posent guère la question du parallèle 
avec l'enquête anthropologique - sans doute parce que l'anthropologie française dont ils se nourrissaient principalement, à la différence de celle qui se développait en Angleterre et aux États-Unis à la même époque, était moins attentive à cette question du statut du terrain et de l'informateur que celle qui s'écrit depuis les années 1990.

Paradoxalement, on pourrait avancer l'idée qu'aujourd'hui le travail de traitement critique des informations recueillies auprès des enquêtés par les anthropologues et le statut donné à ces informations est peut-être plus intéressant pour l'historien du Moyen Âge que la mise en parallèle de résultats d'enquêtes anthropologiques avec des recherches portant sur la société médiévale. La prédominance traditionnelle des sources narratives ou déclaratives dans l'écriture de l'histoire médiévale est en effet une réalité qui mériterait une interrogation plus approfondie. Il ne s'agit pas de faire de ces auteurs du passé l'équivalent des informateurs de l'anthropologue, mais d'utiliser le travail de ces derniers pour s'interroger sur la nature spécifique de ces textes - qui peuvent aussi être relativisés et recontextualisés par la confrontation avec d'autres types de source, mais qui exercent sur l'historien une séduction qui est celle du récit. Le piège est parfois celui de la ressemblance avec la parole vive du témoin, recueillie par l'anthropologue. C'est justement là que les précautions déployées par ce dernier, qui sait à quel point est fragile le statut de cette parole qui a été suscitée, est orienté et doit être resitué socialement et historiquement, sont importantes: si l'anthropologue doit traiter cette parole dont il a pourtant été le témoin direct sans lui donner un statut absolu, combien davantage l'historien, confronté à des textes, c'est-à-dire à l'épaisseur d'une pratique de l'écriture, et à la distance d'une transmission, devra-t-il se montrer attentif aux illusions du "témoignage " et méditer sur la place à donner aux sources et à leur typologie au sein des opérations historiographiques? L'effacement de la procédure d'enquête dans l'écriture de l'histoire, ainsi que la substitution de la critique érudite à une véritable réflexivité deviennent alors de réelles limites de la construction du savoir historique, souvent victime de la séduction du témoignage. Cette attraction est d'autant plus forte que le récit du témoin présente une ressemblance formelle avec la forme d'écriture traditionnelle de l'histoire: dès lors, le choix de certains historiens en faveur d'une construction fragmentaire ou discontinue est le signe d'une rupture, mettant en évidence la position d'enquêteur et d'auteur, et plaçant à distance le témoin sans pour autant $s^{\prime}$ installer en position de surplomb.

9. On peut songer par exemple à l'illusion ethnographique, liée aux sources inquisitoriales, qui colore le livre d'Emmanuel Le Roy Ladurie (1975) et, en contrepoint, aux réserves formulées par Dominick LaCapra (1987), Renato Rosaldo (1986) et John Arnold (2001). 
Ce mouvement n'est pas sans rapport avec la reconsidération du statut du témoin opérée dans l'anthropologie contemporaine. Alors que les dernières années ont plutôt vu se développer la célébration du témoin comme figure principale de l'histoire et interlocuteur de l'historien ${ }^{10}$, l'anthropologie permet de revenir sur le privilège absolu qui lui est conféré, dont on voit bien qu'il a été conçu pour des situations extrêmes, comme le témoignage sur les camps d'extermination, en lui rendant sa dimension pragmatique, à la fois fondamentale dans l'enquête scientifique, et en même temps, contextualisée, socialisée, limitée en un certain sens dans sa validité ${ }^{11}$. En intensifiant la réflexion sur les opérations de production de l'information, sur le statut épistémologique de l'informateur et sur les modalités d'écriture à partir des paroles confiées au chercheur, l'anthropologue et l'historien se rejoignent dans la prise de conscience de la construction méthodologique des liens entretenus avec la documentation - dont l'éloignement chronologique est souvent considéré par l'historien comme une garantie de la neutralisation de son propre engagement ${ }^{12}$.

Dans cette perspective méthodologique, les deux disciplines semblent parfois en situation chiasmatique. Les anthropologues se sont mis à penser leur critique documentaire du terrain en intégrant l'horizon des archives écrites et la structuration critique de celles-ci (Jamin \& Zonabend 2001-2002), tandis que les historiens ont pu être tentés de penser leurs archives comme un "terrain", au sens expérimental, dans la lancée des propositions de la microstoria. Cette approche séduisante a parfois été la source de malentendus, par exemple sur le statut du "réel» dont on s'approcherait et dont on s'éloignerait dans le jeu d'échelles. Le réel de l'historien est d'abord constitué d'archives et d'objets, il n'est pas un terrain réel qui serait le lieu d'une saisie; l'échelle est d'abord une métaphore médiatisée par la structure de la documentation, et c'est vis-à-vis d'elle que la variation s'exerce d'abord (Revel 1996 ; Castelli Gattinara \&

10. Sur le statut du témoin et du témoignage, et leur place dans la réflexion historiographique et épistémologique, cf. par exemple: Annette Wieviorka (1998); Giorgio Agamben (1999); François Hartog (2000) ; Paul Ricoeur (2000 : 201-209, notamment) ; Georges Didi-Huberman (2004) ; Carlo Ginzburg (2010 : 305-334). Les problèmes posés par le roman de Yannick Haenel Yan Karski (2009), et le conflit entre Haenel et Claude Lanzmann relèvent en partie de la même problématique, qui concerne aussi bien l'anthropologue que l'historien; voir sur ce point Patrick Boucheron (2010b).

11. Cf., par exemple, les travaux de Renaud Dulong (1998 et 2009).

12. Pour un exemple méthodologique d'attention au statut du témoin dans le cadre d'une interprétation historique, cf. Alpers \& Hopper (2008). 
Anheim 2009). L'analogie a donc ses limites, mais elle a sa fécondité. Tout d'abord, celle de mettre en évidence le rôle similaire d' «épreuve " joué par le terrain et les archives dans la construction d'un parcours institutionnel d'anthropologue ou d'historien : il faut "faire du terrain " ou "aller aux archives" pour devenir membre de la corporation (Dirks 2002). Ensuite, l'analogie fait signe vers une conception unifiée des sciences sociales dans leur rapport à l'expérimentation: un terrain, sur lequel construire une démarche d'enquête ${ }^{13}$. De ce point de vue, le rapprochement entre anthropologie et histoire a eu un effet inverse à ce qu'on pouvait craindre d'une indifférenciation progressive : la convergence autour d'un rapprochement épistémologique, liée à la distinction d'origine disciplinaire, fait qu'il n'a jamais été aussi intéressant qu'aujourd'hui pour un historien de lire de l'anthropologie pour saisir la nature des opérations cognitives et documentaires en lien avec sa propre pratique. Cela nécessite sans doute un effort de transposition plus important que celui qui consistait à l'importation d'objets ou de modèles, reposant sur une décomposition plus abstraite des mécanismes de l'élaboration scientifique, mais l'enrichissement est à la mesure de l'effort à fournir.

En intégrant le «temps du document» aux procédures d'enquête, l'anthropologie s'est inscrite dans un déplacement qui dépasse la seule réflexion sur le statut des "sources" et qui a aussi eu pour conséquence de mettre, à la différence de la tradition de l'anthropologie structurale, la discipline face à sa propre historicité. À des opérations savantes qui consistaient à effacer l'historicité des informations recueillies et leur contextualité, mettant par exemple sur le même plan les enquêtes orales et les récits anciens concernant les populations étudiées afin de produire un modèle clos d'interprétation, ont succédé de nouvelles démarches interprétatives, rappelant que les récits des explorateurs et des missionnaires étaient eux-mêmes enserrés dans une historicité modelée par la rencontre entre les Occidentaux et les non-Occidentaux, et que les peuples prétendument sans histoire en avaient bien une (outre les travaux de Terray, Adler et Izard notamment, déjà cités, cf. Naepels, ed. 2010). En amont de l'intervention de l'anthropologue s'est donc ouvert un champ d'investigation scientifique profondément ancré dans l'historicité,

13. On pourra placer, dans cette perspective, les recherches menées depuis près de vingt ans au sein de la collection Enquête, ainsi que les réflexions exposées par Jean-Claude Passeron (2005 [1991]), qui critique le parallèle entre statut de l'expérimentation dans les sciences de la nature et dans les sciences de l'homme, mais qui fait de la référence empirique, sous la forme par exemple de la thématique de l'indexation, l'un des éléments centraux d'une théorie unificatrice réunissant histoire, anthropologie et sociologie. 
ce qui a modifié non seulement la place des pratiques historiennes au sein de l'enquête anthropologique, mais même la saisie de la société sur le vif. De manière à ne pas répéter l'absoluité d'une situation singulière qui avait pu contribuer à prendre les récits missionnaires pour une vérité générale, les anthropologues sont désormais attentifs aux contextes historiques d'énonciation de l'oralité qu'ils peuvent recueillir (Bazin 2006 ; Jolly 2010). C'est donc l'ensemble de la démarche anthropologique qui est ouverte non seulement aux méthodes des historiens, mais aussi à l'historicité au sens le plus radical, d'autant plus qu'en aval de l'opération anthropologique, comme le constatent les anthropologues à chaque fois qu'ils retournent sur leur terrain, l'historicité est omniprésente: les sociétés étudiées changent à vue d'œil et il est impossible de ne pas en tenir compte dans la pratique scientifique elle-même.

Ces modifications dans la perception historique du terrain ont rendu les anthropologues aussi conscients des enjeux de l'historicité pour leur pratique scientifique que le sont les historiens, ce qui crée une véritable convergence. La complexité des temporalités, de leur feuilletage, de leur superposition et de leur retour est devenue un problème théorique traité par les anthropologues et un enjeu majeur, parfois même davantage qu'il ne l'est pour les historiens. La timide introduction de la référence aux travaux de Reinhardt Koselleck (1990, 1997), le succès de la notion de "régime d'historicité" dans la lancée du livre de François Hartog (2002), notion elle-même élaborée dans un dialogue avec l'anthropologue Gérard Lenclud, et les travaux sur la temporalité comme ceux de Jacques Le Goff (1977) et Jean-Claude Schmitt (2001, 2010b) n'ont eu qu'une influence limitée sur la pratique des historiens. La temporalité, qui figure en bonne place dans la discussion à l'origine du «tournant critique ", est cependant restée l'un des angles morts de l'historiographie (cf. Annales. ESC 1989). Parfois, les historiens affirment travailler sur le temps, alors qu'ils plongent la société et les documents qu'ils étudient dans une fiction temporelle linéaire faisant appel à une conception simplifiée de la causalité et de la consécution, en gardant chevillée au corps la croyance selon laquelle le principal problème de l'historien est l'anachronisme. Les études historiques ne prennent pas toujours au sérieux la question de l'historicité de la société qui est étudiée et l'arsenal conceptuel se réduit dans certains cas à des couples de notions telles que "rupture et continuité ", alors que la question est posée par des anthropologues comme Johannes Fabian (2006 [1983]) ou Nicholas Thomas (1998 [1989]), même si leur réception n’a pas été généralisée non plus au sein de leur propre discipline, à l'exception des remarques et critiques de Marc Augé (1994). 
La question est centrale dès lors qu'on cherche à trouver des outils pour penser la transformation sociale, en particulier quand elle est directement liée à une transformation des cadres de perception de la temporalité. C'est le cœur même du problème posé par le concept de "Moyen Âge ", défini rétrospectivement comme un temps d'entre-deux, une époque intermédiaire. Le médiéviste hérite d'une difficulté méthodologique constituée par l'historiographie depuis le XVI $I^{\text {e }}$ siècle : comment penser positivement la dynamique historique et sociale d'un moment temporel dont l'identité est négative et construite de l'extérieur? Cette question n'est pas si différente de celle posée par la tradition anthropologique, qui s'appuie sur une discipline consacrée à l'altérité de l'Occident contemporain - ce qui explique sans doute certaines des affinités entre médiévistique et anthropologie. Dès lors, les efforts accomplis par certains anthropologues depuis les années 1980 pour «synchroniser » la temporalité des chercheurs et celle des sociétés étudiées intéressent à double titre l'historien du Moyen Âge, en particulier quand il s'aventure aux confins de celui-ci et des débuts de la modernité, du côté de ce qu'on appelle conventionnellement la «Renaissance».

La notion de "co-temporalité » élaborée par Johannes Fabian peut ainsi être entendue par l'historien en deux sens. Tout d'abord, comme rappel du fait que les hommes de la Renaissance et ceux du Moyen Âge finissant sont les mêmes: qu'ils partagent, concrètement, la même temporalité et que, contrairement aux lieux communs sur l'« esprit médiéval » et l'« esprit renaissant", les mêmes hommes sont tour à tour et en même temps, selon nos critères, "médiévaux " et "renaissants ". Les transformations de l'Occident entre le XIII ${ }^{\mathrm{e}}$ et le XVI ${ }^{\mathrm{e}}$ siècle ne peuvent pas se penser sans des catégories de temporalité complexes, qui brisent la linéarité stricte: comme Roger Bastide (1955, 2001 [1958]) l'avait montré pour le Brésil avec le "principe de coupure» sans beaucoup intéresser les historiens, comme Johannes Fabian (2006 [1983]) l'a théorisé dans une perspective liée aux problématiques postcoloniales, les acteurs sociaux sont pris dans un enchevêtrement de temporalités et de cultures présentes simultanément: c'est sans doute la complexification de cet écheveau de temps superposés qui caractérise ce moment chronologique de la Renaissance durant lequel la société occidentale se transforme tout en transformant son propre cadre de perception temporel, recréant la fiction d'une filiation directe avec l'Antiquité et refoulant un millénaire qui ne disparaît pas pour autant mais dont la présence fait sans cesse retour, d'une manière devenue moins visible ${ }^{14}$. Il ne s'agit pas de dire que rien n'a changé en

14. Sur cette question complexe, il faut considérer l'importance d'un héritage historiographique qui a contribué, depuis le XIX $X^{\mathrm{e}}$ siècle, à relayer la vision des auteurs qui ont participé à la .../...

\section{Étienne Anheim}


Occident $\mathrm{du}$ XIII ${ }^{\mathrm{e}}$ au XVI ${ }^{\mathrm{e}}$ siècle, mais plutôt de réfléchir au fait que ce changement n'a pas été simplement une affaire de rupture et de continuité, mais aussi de remaniement et de superposition de temporalités, à la manière dont les anthropologues cherchent à les penser dans les sociétés non occidentales intégrées au flux de la mondialisation occidentalisée de nos jours.

Mais la "co-temporalité " de Johannes Fabian possède aussi une autre dimension pour l'historien : celle qui consiste à lui faire considérer avec distance son propre imaginaire, qui le conduit souvent à se représenter comme partageant la même temporalité que certains acteurs du passé, et au contraire, comme radicalement séparé d'autres. Dans le domaine des études renaissantes, combien d'historiens se considèrent-ils de plain-pied avec les valeurs qu'ils baptisent "humanistes", tandis qu'ils se voient comme radicalement éloignés de celles des médiévaux "scolastiques" - ou, inversement, puisque l'histoire de la scolastique est désormais bien installée dans le paysage universitaire? Cette "cotemporalité sélective » rappelle que l'historien lui-même crée inconsciemment son propre ordonnancement du temps, qui n'est pas linéaire non plus, mais qui affecte profondément sa recherche: le rappel de Fabian à ses collègues peut aussi être entendu par les historiens. Ils ne sont les contemporains d'aucun homme du passé, entendu de manière radicale, et ils partagent en revanche la temporalité de l'ensemble des vestiges du passé qui sont à leur disposition - et il n'y a pas de raison méthodologique d'en privilégier certains plutôt que d'autres. Ces enjeux touchant à la temporalité sont fondamentaux et dépassent la question de l'anthropologie, qui rejoint ici l'histoire de l'art, de Warburg à Didi-Huberman (2002), ou la sociologie, en particulier celle de Maurice Halbwachs (1994 [1925], 1997 [1950], 2008 [1941]) : s'élabore progressivement, comme l'a pointé Éric Brian (2009) dans le contexte de la science économique, un "nouvel historicisme», parfois aux frontières de la discipline historique, mais dont les historiens peuvent s'inspirer avec profit.

[Suite de la note 14] fabrication de l'idéal "renaissant", et qui a largement participé, par la construction chronologique des périodes et la division institutionnelle universitaire, à donner une consistance au "Moyen Âge» : cf., par exemple, Burckhardt (2008 [1860]) ou Huizinga (2002 [1919]). Pour une approche historiographique, cf. : Ferguson (2009 [1948]) ; Vöss (1972); Fubini (2001). 


\section{Jeux d'identité : le sujet et l'objet}

Cette réintroduction de l'historicité peut aussi faire apparaitre aux yeux de l'historien des questions qui concernent son identité savante et son rapport avec les sociétés qu'il étudie, alors que ces questions, dans sa pratique disciplinaire quotidienne, restent en général enfouies. Pendant longtemps, l'anthropologie a servi aux historiens à produire un effet de distanciation en produisant de l'«autre». Dans le cas des sociétés anciennes et médiévales en particulier, l'anthropologie a eu pour effet d'arrêter le temps, comme l'a bien montré Nicole Loraux (2005) ${ }^{15}$, et d'éloigner des sociétés qu'on aurait facilement tenues pour voisines, parce qu'elles nous sont antécédentes dans l'ordre de la culture ${ }^{16}$. L'anthropologie a permis aux historiens de rendre étranger ce qui était apparemment familier et, inversement, de rendre familier ce qui était étranger. Mais cette pratique a parfois donné lieu à un "ensauvagement " qui mettait l'accent sur une coupure absolue entre l'historien et la société passée qu'il étudiait. Sans doute était-ce là le prix à payer pour un vrai gain d'intelligibilité ; cependant les évolutions récentes de l'anthropologie peuvent donner les moyens de revenir sur cette coupure radicale mais fictive.

En effet, dans la pratique anthropologique, il n'y a jamais de séparation totale entre le point de vue du chercheur et celui de l'"indigène», dès lors qu'ils sont en interaction et, plus encore, en recherche d'intercompréhension, donc engagés dans un processus de traduction. Le lien noué au cours de l'enquête anthropologique a pour effet l'impossibilité d'une césure absolue, dont on peut examiner les conséquences. Lorsqu'on étudie l'histoire du Moyen Âge, l'une des difficultés posées par l'importation de concepts anthropologiques traditionnels est qu'ils révèlent souvent leurs limites précisément dans les situations où la société médiévale se spécifie comme une société proprement occidentale, par exemple dans la structure juridico-institutionnelle d'héritage romain ou dans les usages et les pratiques de l'écrit : souvent, les analyses inspirées d'études anthropologiques sur les rituels ou l'oralité, si elles sont utiles pour rappeler que le Moyen Âge n'est pas la modernité occidentale, échouent en même temps à en rendre complètement compte ${ }^{17}$. L'historien médiéviste semble

15. Par exemple : «L'acte inaugural de l'anthropologie de la Grèce semble être d'arrêter le temps civique [...]. De cette immobilisation résulte évidemment la possibilité de généraliser " (Loraux 2005: 45); ou : « [...] l'anthropologie de la Grèce a de fait "refroidi” l'objet cité, mettant du même coup sur le devant tout ce qui apparente cette forme politique à une société froide " (Ibid.: 54).

16. Ce n'est pas un hasard si la thématique de l'«estrangement" a été développée par l'un des historiens les plus proches de l'anthropologie, Carlo Ginzburg (2001).

17. Sur la question du rituel, cf. par exemple les observations critiques de Philippe Buc (2003). 
parfois condamné à une alternative impossible: ou bien moderniser le Moyen Âge, ou au contraire l'ensauvager, sans qu'aucune des deux postures ne soit vraiment convaincante. C'est aussi ce qui fait son intérêt intellectuel : la singularité scientifique des sociétés occidentales anciennes et médiévales tient en effet principalement à ce qu'elles entretiennent avec le chercheur un double lien. Il s'agit de sociétés radicalement différentes de la nôtre, dont l'étude nécessite un dépaysement apparemment aussi grand que celle de sociétés très éloignées de l'Occident dans l'espace. Mais, en même temps, ce sont des sociétés qui ont avec notre présent un air de famille, d'antécédence chronologique certes, mais surtout de généalogie, et qui continuent à exister non pas seulement à travers notre travail d'historiens, mais aussi à travers notre vie quotidienne d'individus modernes (Morsel 2007 ; Anheim 2008b). Mettre au centre de sa recherche la transformation des sociétés "anciennes" en sociétés "modernes", comme le font implicitement beaucoup d'historiens et de praticiens des sciences sociales depuis Marx, Weber ou Fustel de Coulanges, revient par conséquent à poser la question du moment de jonction chronologique entre le point de vue de l'enquêteur et celui de l'enquêté. Il s'agit de poser explicitement une question qui est tacitement à l'arrière-plan de bien des travaux d'historiens: quand supposons-nous être devenus "modernes» - et que désigne ce "nous" ?

Prenons l'exemple des pratiques musicales savantes du Moyen Âge. Leur analyse selon les critères traditionnels de l'histoire de la musique occidentale est difficile dans la mesure où c'est la notion même de "musique", en tant que sphère autonome de pratiques sonores liées à un usage social et esthétique qui fait défaut. Les recherches des ethnomusicologues sont à cet égard beaucoup plus utiles pour comprendre la place de la polyphonie liée à la liturgie, ce qui témoigne de l'apport fondamental de l'anthropologie (Aubert 2010 ; Fritz 2000). Mais, dans le même temps, la particularité de cette musique est précisément qu'elle est liée à la naissance d'une sphère singulière qu'on appelle "musique " au sens moderne. C'est justement au cours du Moyen Âge que s'inventent les cadres sociaux, théoriques et musicologiques permettant la construction de la musique occidentale classique, qui elle-même a nourri l'éducation des historiens qui vont ensuite travailler sur les pratiques musicales médiévales (Colette 2008 ; Anheim 2000, 2008a). Face à cette situation, qui est l'un des problèmes les plus couramment posés au médiéviste lorsqu'il cherche les outils adaptés à la saisie de son objet dans le trousseau des sciences sociales, la thématisation par l'anthropologie contemporaine des liens entre enquêteur et enquêté est précieuse. 
Certes, l'historien n'est pas en situation d'interaction, sinon dans l'histoire du temps présent, ce qui empêche toute transposition directe de la situation anthropologique. Cependant, l'influence réciproque qui s'exerce au cours de l'enquête et des échanges peut servir de point de repère pour penser la nature du lien généalogique qui unit les historiens aux sociétés qui l'ont précédé, en particulier quand il s'agit des sociétés héritées du monde gréco-romain. C'est ce que suggère l'«anthropologie des savoirs" telle que la pratique Wiktor Stoczkowski $(2007,2008)^{18}$. Les concepts de l'anthropologie sont en effet nés dans des contextes dont les anthropologues, à condition d'une enquête mobilisant des documents traditionnellement utilisés par les historiens, peuvent rendre compte. Ce faisant, l'anthropologue peut inscrire ses propres concepts dans une historicisation radicale qui engage une généalogie. Au travers de schémas intellectuels hérités et parfois très anciens, l'anthropologie, comme les autres sciences sociales, porte elle-même la trace de l'histoire de la société qui lui a donné naissance, de sorte que, quand elle retourne ses outils sur le passé de cette société, elle ne fait parfois que la refléter en miroir. Mais il arrive que le miroir s'inverse : ainsi, la catégorie du "don", appliquée par Marcel Mauss aux sociétés traditionnelles, puis transposée à l'histoire ancienne ou médiévale, s'est-elle construite comme l'envers de l'échange marchand de l'Occident moderne, mais en même temps, elle reconduit en l'inversant une appréhension moderne du monde, et ne peut se comprendre sans référence à la culture littéraire et religieuse des individus qui l'ont forgée ${ }^{19}$. Derrière la surprise éprouvée par certains historiens de voir fonctionner des concepts anthropologiques appliqués à des sociétés occidentales pour lesquels ils n'avaient pas été conçus, se cache parfois un peu d'ingénuité à l'égard de la construction de ces concepts. Lanthropologie des savoirs, qui aide à la dissiper, permet la mise en œuvre d'enquêtes historiques réflexives conscientes de ce double lien avec un passé qui détermine le chercheur dans ses effets lointains, et qui ne peut donc pas être saisi dans la fiction d'une coupure radicale.

Les lectures anthropologiques permettent aussi d'explorer les conséquences de l'effondrement de la «frontière" entre l'Occident et son Autre, qui a pourtant été fondamentale tant dans la genèse de l'anthropologie comme discipline que dans le développement de l'anthropologie historique comme programme de recherche. Ce que montre

18. On peut rapprocher cette démarche du programme d' "anthropologie scolastique " formulé par Alain Boureau qui, lui aussi, s'appuie sur un usage intensif de la réflexivité pour lire les documents médiévaux, ou de l'approche illustrée par Dominique Iogna-Prat (2006).

19. Pour un aperçu des enjeux historiographiques de la catégorie de Mauss, au sein d'une innombrable bibliographie, cf., en particulier, Magnani (2007) et Azoulay (2012, à paraître). 
l'anthropologie récente, de même que beaucoup de courants historiques nourris du dialogue avec les colonial studies, comme l'" histoire connectée ", c'est que la société tenue complètement à l'écart de l'occidentalisation est définitivement devenue un mythe et que la pureté du point de vue interne est une illusion. Il y a toujours un niveau d'interaction minimal tel, qu'une analyse purement "indigène" nécessiterait l'élimination rétrospective par le chercheur des transformations historiques à l'œuvre et des échanges qui modifient à chaque moment la position des interlocuteurs. L'anthropologie est relationnelle, pour le pire et le meilleur, et offre ainsi un moyen de penser la relation de l'historien avec la société qu'il étudie d'une manière renouvelée.

En effet, l'idée que l'historien travaille sur le passé relève d'une illusion, on le sait depuis au moins Walter Benjamin et Marc Bloch : il travaille sur la présence et la prolongation du passé dans le présent, à travers une multitude de signes, de traces, textuelles ou non, ou d'objets. Comme l'anthropologue, l'historien est, vis-à-vis de son objet, en situation de coprésence, même si elle n'est pas dialogique : le passé est là, et cette présence est un lien qu'il est nécessaire de penser - plus radicalement, le passé est dans l'historien lui-même, sédimenté sous la forme d'une culture qui oriente des choix épistémologiques d'une manière parfois difficile à percevoir.

Le refoulement de ce lien n'aboutit qu'à créer une fiction de distance, là où une distanciation scientifique nécessiterait d'abord la reconnaissance d'une proximité. L'une des difficultés fondamentales de l'historien de l'Occident est l'hypothèque téléologique, qui s'exerce plus fortement à mesure qu'on travaille sur une société qui est davantage dans un rapport généalogique direct avec la sienne: non seulement on connaît la fin de l'histoire, mais, de plus, "nous " sommes, directement, la fin de cette histoire. Difficile, dès lors, de faire comme si cela n'existait pas. Pourtant, de quels outils dispose l'historien pour penser cette implication complexe dans son objet d'étude ? Il n'en a guère et, si l'arsenal théorique de l'historien est devenu extrêmement raffiné dans le domaine de la construction d'objet ou de l'épistémologie de la connaissance, on peut noter la rareté des réflexions sur les conséquences de ce rapport au passé qui implique l'historien en tant qu'individu et produit d'un devenir historique.

Il est difficile d'échapper aux implications de ce problème, même lorsque le choix d'une approche anthropologique structurale continue à être revendiqué. C'est le cas du livre de Philippe Descola Par-delà nature et culture (2005) et de ses discussions par les historiens. L'approche de Descola se revendique de la poursuite du modèle structural et il propose une division en quatre des formes de rapport au monde qui s'inscrit dans la lignée de cet héritage, mais, en même temps, l'insertion de l'Occident 
comme l'un des quatre types possibles produit un effet paradoxal, assumé par l'auteur, celui d'une historicisation de l'ensemble du livre. La mise en parallèle d'un modèle "naturaliste " occidental avec d'autres modèles extra-européens contribue ainsi à rendre compte de l'étrangeté anthropologique de notre coupure entre nature et culture, tout en posant un double problème, celui de l'historicité de cette conception, et celui de sa progressive extension, par le biais de la domination occidentale, à l'ensemble du monde. C'est ainsi que l'ouvrage a été reçu et discuté au sein des études médiévales (Coste 2010; Anheim 2008b) : c'est moins l'héritage structural que l'intégration d'une problématique historique liée au rapport entre l'Occident et le reste des sociétés qui a donné à ce livre un écho dans le travail de certains médiévistes, confirmant paradoxalement l'évolution que j'essaie de décrire à propos des intérêts anthropologiques des historiens.

Il s'agit finalement de trouver dans l'anthropologie les outils pour l'articulation des temporalités et, plus largement, l'articulation des mondes, entre l'Occident et son - ou plutôt ses Autres - ou l'Occident et son passé. Ce faisant, c'est la position du sujet savant qui est déplacée. L'historien, même après la rupture antipositiviste des Annales, a souvent gardé l'habitude de se penser comme un sujet absolu, radicalement séparé de son objet - refusant en général de thématiser les liens généalogiques, affectifs ou politiques l'unissant à ses objets, ou au contraire les confessant avec une certaine candeur militante ou sentimentale tout en se réfugiant derrière des précautions de méthode pour justifier la scientificité de son travail. L'un des apports de l'anthropologie est de montrer la vanité de cette position, et de rappeler l'engagement du chercheur en sciences sociales dans le même monde que ses objets (outre l'œuvre emblématique d'un Michel Leiris [1996], voir: Weber [2008] ; Naepels [1998, 2011]). Cela ne veut pas dire qu'il n'y aurait pas une construction différenciée possible de la connaissance au sein de ce monde, ou bien que le chercheur serait condamné à un relativisme sans issue, mais que le déni de cet " engagement", pour rappeler la terminologie de Norbert Elias (1993), est la pire des solutions.

Depuis longtemps, la tradition anthropologique s'est engagée de ce point de vue dans une direction différente de celle des historiens, ce qui est d'abord le résultat de la présence physique du chercheur parmi ceux qu'il étudie. Dès lors que le terrain et la rencontre sont placés au cœur du métier, la présence est un élément fondamental de la connaissance. Cette dimension est en revanche en général effacée du travail de l'historien : son immersion dans la documentation ancienne est considérée comme une activité neutre, ce qui est loin d'être démontré. N'y aurait-il 
rien à objectiver du désir de savoir de l'historien, là où celui de l'anthropologue semble au contraire constituer un élément fondamental de l'enquête, exposé au regard de la communauté scientifique ? En corollaire, l'exposition du processus de fabrication de la recherche tient une place importante dans la restitution des données de l'anthropologue, ce qui n'est que très rarement le cas chez les historiens. Ces derniers considèrent avec naturalité l'essentiel des opérations effectuées sur la documentation (Debaene 2010). Là où l'anthropologue, parce qu'il a affaire à des personnes, déploie souvent un luxe de précautions et de contrôle réflexif, l'historien se contente souvent de son sens commun dans des investigations qui ne sont pas moins délicates et qui, finalement, ne laissent à peu près aucune trace dans l'écriture historique. Car, tandis que l'anthropologue n'hésite pas à se mettre en scène dans l'écriture, non pas par souci d'affirmation individuelle, mais par soin de montrer son intervention directe dans l'élaboration de ce savoir - même si la démonstrativité du témoin de retour de son terrain semble parfois excessive au lecteur -, l'historien se gomme soigneusement, hormis lors de quelques récits alors centrés sur lui et non sur la construction de son objet historique ${ }^{20}$. Pourtant, la mise en évidence des procédures de l'enquête, par lesquelles l'historien parvient à construire son objet en intervenant directement dans son élaboration, n'est pas moins essentielle et intéressante dans son domaine, comme le montrent de récents exemples pour l'histoire du Moyen Âge.

Le livre de Jacques Dalarun (2007), présente ainsi un usage original d'une érudition extrêmement classique : plutôt que de considérer le travail philologique du chercheur comme une phase préparatoire au récit historique, il est ici intégré dans ce récit qui met en scène les procédures concrètes de traitement de l'information par l'historien, en l'occurrence le travail sur les documents franciscains du XIII siècle concernant la vie de François. Puis, dans une seconde phase, second choix narratif spécifique, l'auteur, au lieu de proposer une interprétation des documents étudiés, propose trois récits parallèles qui seraient trois manières de mettre ensemble les pièces du puzzle. Venant d'un auteur qui a toujours donné une place dans ses travaux à la subjectivité de l'historien et à l'exposition de ses méthodes de collecte de la documentation, le choix ne surprend

20. Le livre le plus célèbre dans cette perspective est celui dirigé par Pierre Nora (1986), dont l'orientation dominante n'est pas réflexive, mais plutôt classiquement autobiographique. La sensibilité réflexive des historiens vis-à-vis de leur parcours tend cependant à se développer, sous l'influence du mémoire de synthèse de l'HDR, comme en témoigne par exemple Patrick Boucheron (2010b), inaugurant une collection destinée à la publication de textes de ce genre, mais aussi, dans un cadre plus libre, comme le montrent Michel Pastoureau (2010) et Alain Boureau (2011). 
pas (1992). Même s'il joue parfois avec la limite - les trois récits ne sont pas également convaincants -, il montre le travail scientifique à l'œuvre au sein même du livre. Au même moment, Didier Lett écrit Un procès de canonisation au Moyen Âge. Essai d'histoire sociale (2008), dans lequel c'est également le traitement par l'historien de son document, un procès de canonisation, qui devient le fil conducteur du récit, ce qui met le lecteur dans la situation d'avoir une vision panoramique de la procédure de recherche et non simplement des résultats (Ramage 2009). C'est enfin la même démarche qui est choisie par Joseph Morsel dans un mémoire d'habilitation, encore inédit, présenté en 2009 et portant sur un corpus de lettres du début du XVI ${ }^{\mathrm{e}}$ siècle analysé principalement sous l'angle de sa constitution, de sa transmission et de son usage, et non pour les informations positives qu'il permet d'apprendre, soulignant que l'enjeu fondamental de l'écriture de l'histoire relève moins pour lui de la communication des résultats que de la communication des procédures scientifiques utilisées par le chercheur et permettant de parvenir à tel ou tel résultat. Au-delà des nombreuses différences entre les approches de ces trois historiens, se dessine ainsi une convergence qui n'a rien de fortuite: il s'agit bien d'une réévaluation dans la forme même de la narration de la pratique de recherche de l'historien, qui inscrit ainsi explicitement sa présence dans l'écriture historique.

Les implications éthiques de cette présence du sujet savant méritent également l'attention. Les complexes cas de conscience des anthropologues qui sont aussi ceux des sociologues, la nécessité de faire disparaître l'identité de leurs informateurs, ou bien de ne pas livrer finalement des éléments qui pourraient avoir en retour un effet négatif sur leur terrain d'investigation, sont des problèmes qui peuvent faire réfléchir les historiens. Il s'agit d'une manière éthique de poser la question de la restitution des paroles ou des informations que détient le chercheur. Aux réactions parfois négatives ou hostiles des individus rencontrés au cours de l'enquête de terrain pourrait correspondre le développement des attaques contre les historiens de la part d'associations mémorielles de descendants de tels ou tels groupes d'acteurs historiques ${ }^{21}$. C'est à ce point

21. On pourra bien sûr songer, du côté des historiens, à l'affaire Pétré-Grenouilleau, aux mises en cause diverses d'historiens dans un cadre judiciaire ou journalistique, depuis les années 1990 au moins, ou encore, dans une autre perspective, aux réclamations de pays ou d'institutions muséales étrangers pour la restitution d'objets désormais exposés en Occident, souvent avec la complicité des historiens et des anthropologues, cf. Benoît de l'Estoile (2007). Ces questions sont déstabilisantes pour les historiens d'autant plus qu'elles sont relativement nouvelles, tandis que les anthropologues, comme les sociologues, ont intégré le problème depuis un certain temps, y compris dans la pratique de terrain, sous la forme par exemple de codes d'éthique (cf. : Cefaï, ed. 2010 ; Bensa \& Fassin 2008 ; et Laurens \& Neyrat 2010). 
que pourrait intervenir le "souci des morts»: ce n'est pas parce que les individus sur lesquels travaille l'historien ont le plus souvent disparu que ce dernier ne peut pas se poser des questions d'éthique scientifique de ce genre (ce qui ne veut pas dire se soumettre aux revendications mémorielles) lorsqu'il utilise, critique, analyse et met en scène les mots du passé, plutôt que de s'interroger sur son éventuelle «dette » en termes spiritualistes.

Bien des éléments mis en évidence dans le rapport entre histoire et anthropologie relèvent de la convergence, qu'on aurait aussi pu retrouver avec certains courants de la sociologie, plus que de l'influence. Le propos n'est pas de considérer que l'historien doit se mettre à l'école d'une nouvelle anthropologie, ou l'inverse. Mais, si l'anthropologie continue à enrichir les enquêtes empiriques des historiens ${ }^{22}$, voici aussi ce qu'elle peut lui apporter: une reconsidération de sa position savante, ainsi que des rapports complexes entre son activité, composée d'enquête, de désir, d'écriture, et la vie propre de ce qu'il étudie, qu'il s'agisse de gens ou d'un passé qui n'a de sens que parce qu'il est encore présent sous la forme de traces matérielles, mais aussi d'un héritage inscrit dans le corps et l'esprit mêmes du chercheur. L'anthropologie, historiquement, s'est construite comme un savoir sur l'autre de l'Occident, dans l'espace. C'est ainsi que les historiens l'ont utilisée, pour en faire un savoir sur l'autre dans le temps, même si cet usage s'est parfois retourné contre eux en les enfermant dans l'alternative impossible des anciens contre les modernes. L'effort pour penser la temporalité produit par l'anthropologie depuis les années 1980 est l'occasion pour les historiens d'écarter certaines de ces apories: de même qu'il y a co-temporalité entre l'anthropologue et les « indigènes ", il y a co-temporalité entre l'historien et sa documentation qui a été préservée et a survécu jusqu'à lui en tant que partie toujours réactualisée du passé. Lorsque l'historien analyse le changement historique, il pose

22. Même si ce n'était pas l'objet de ce travail, et outre les féconds prolongements de l'anthropologie historique déjà évoqués au début de cet article, il faut rappeler les fructueux échanges empiriques que les historiens, en particulier pour le Moyen Âge, ont pu avoir depuis vingt ans avec différents courants de l'anthropologie, qu'il s'agisse par exemple de l'anthropologie juridique dans le cadre des débats sur la mutation de l'an mil (cf. les travaux de Dominique Barthélemy [1997, 1999]), de l'anthropologie visuelle de chercheurs comme Hans Belting (cf. Schmitt [2002]; ou Baschet [2008]), de l'anthropologie des pratiques d'écriture dans la lignée de Jack Goody (pour une présentation synthétique de l'historiographie médiévale dans ce domaine, cf. Chastang [2008]), de l'anthropologie économique (avec Feller, Gramain \& Weber [2005]), ou encore des réflexions de Maurice Godelier ou de Louis Dumont (mobilisés par Iogna-Prat [1998, 2006]). 
toujours un "avant» et un "après ", comme l'anthropologue pose un "ici» et un "ailleurs». Cette fiction peut être productrice de savoir, à condition de ne pas oublier qu'il s'agit d'une fiction. L'anthropologue rappelle à l'historien qu'il est engagé dans un lien avec l'objet de son travail, même s'il s'agit d'archives, de manuscrits ou de vestiges archéologiques. L'«ailleurs» et l'«avant» sont toujours, pour l'un et l'autre, des «maintenant » qui engagent la subjectivité, quelles que soient les raisons qui en justifient la présence ou la rémanence. Peut-être la traversée du miroir n'était-elle qu'un rêve, de même que ce pays exotique que les historiens découvraient en suivant les anthropologues. Aujourd'hui, l'anthropologie n'est plus le pays des merveilles de l'historien; il n'y a rien au-delà du miroir. Face à lui, les historiens et les anthropologues sont côte à côte. Mais, à ce stade, il arrive qu'on se connaisse mieux en regardant le reflet de son voisin : soi-même comme un autre.

Université de Versailles - Saint-Quentin-en-Yvelines Laboratoire États, Société, Religion (ESR), Institut d'études culturelles, Versailles valetien@gmail.com

MOTS CLÉS/KEYWORDS : histoire et anthropologie/history and anthropology - Moyen Âge/ Middle Ages - sources - documentation - subjectivité/subjectivity - témoignage/evidence. 
Adler, Alfred

1982 La Mort est le masque du roi. La royauté sacrée des Moundang du Tchad. Paris, Payot.

\section{Agamben, Giorgio}

1999 Ce qui reste d'Auschwitz.

Paris, Rivages.

\section{Albert, Jean-Pierre}

2010 «L'histoire et l'anthropologie : convergences et spécificités ", in Elisa Brilli, Blaise Dufal \& Pierre-Olivier Dittmar, eds, Ateliers du centre de recherches historiques $6 \ldots$ [http ://acrh.revues.org/1944].

\section{Alpers, Edward A. \& Matthew S. Hopper}

2008 «Parler en son nom? Comprendre les témoignages d'esclaves africains originaires de l'Océan Indien

(1850-1930)", Annales. HSS 63 (4) : 799-828.

\section{Anheim, Étienne}

2000 «Une controverse médiévale sur la musique : la décrétale Docta sanctorum (1324-1325) de Jean XXII et le débat sur l'ars nova dans les années 1320 ", Revue Mabillon nouv. sér. 11 : 221-246, 2008a «La musique polyphonique à la cour des papes au XIVe siècle : une sociologie historique ", Bulletin du centre d'études médiévales d'Auxerre 2 [http ://cem.revues.org/index9422.html]. 2008b «La Chambre du Cerf: image, savoir et nature à Avignon au milieu du XIV siècle", Micrologus $16: 57-124$.

2010 «Art, littérature et anthropologie historique : quelques réflexions historiographiques ", in Elisa Brilli, Blaise Dufal \& Pierre-Olivier Dittmar, eds, Ateliers du centre de recherches historiques $6 \ldots$ [http ://acrh.revues.org/index2818].

\section{Anheim, Étienne \& Olivier Poncet}

2004 «Fabrique des archives, fabrique de l'histoire ", Revue de synthèse 125 : 1-14.
Annales. EsC

1989 Annales. Économies, Sociétés,

Civilisations 44 (6) : Histoire et sciences

sociales, un tournant critique.

Paris, Armand Colin.

Arnold, John $\mathrm{H}$

2001 Inquisition and Power. Catharism and the Confessing Subject in Medieval Languedoc. Philadelphie, University of Pennsylvania Press.

\section{Aubert, Eduardo Henrik}

2010 "L'anthropologie historique par le détour de la musicologie : une ethnomusicologie historique du Moyen Âge est-elle souhaitable? ", in Elisa Brilli, Blaise Dufal \& Pierre-Olivier Dittmar, eds, Ateliers du centre de recherches historiques $6 \ldots$ [http ://acrh.revues.org/1916].

\section{Augé, Marc}

1979 Symbole, fonction, histoire.

Les interrogations de l'anthropologie.

Paris, Hachette.

1994 Pour une anthropologie des mondes contemporains. Paris, Aubier.

\section{Azoulay, Vincent}

2012 «Du paradigme du don à une anthropologie pragmatique de la valeur", in Pascal Payen \& Évelyne Scheid, eds, Antiquité et anthropologie. Bilans et perspective. Actes des journées d'étude internationales de Toulouse (18-19 mars 2010). Turnhout, Brepols [à paraître].

\section{Barthélemy, Dominique}

1997 La Mutation de l'an mil a-t-elle eu lieu? Paris, Fayard.

1999 L'An mil et la paix de Dieu.

Paris, Fayard.

Baschet, Jérôme

2008 Iconographie médiévale.

Paris, Gallimard

(«Folio. Histoire» 161). 


\section{Bastide, Roger}

1955 «Le principe de coupure

et le comportement afro-brésilien ",

Anais do $31^{e}$ Congresso Internacional

de Americanistas, Sao Paulo (1954).

Sao Paulo, Anhembi : 493-503.

2001 [1958] Le Candomblé de Bahia.

Rite Nagô. Paris, Plon

("Terre humaine»).

Bazin, Jean

2006 Des clous dans la Joconde.

L'anthropologie autrement.

Toulouse, Anacharsis.

Bensa, Alban

2006 La Fin de l'exotisme.

Essais d'anthropologie critique.

Toulouse, Anacharsis.

Bensa, Alban \& Didier Fassin

2008 Les Politiques de l'enquête.

Épreuves ethnographiques.

Paris, La Découverte.

Bonhomme, Julien

2005 Le Miroir et le crâne.

Parcours initiatique du Bwete misoko.

Paris, CNRS Éd.

2009 Le Voleur de sexe. Anthropologie

d'une rumeur africaine. Paris, Le Seuil.

Boucheron, Patrick

1998 "Saint Louis, comédien et martyr :

l'écriture d'une vie", Médiévales 34: 69-77

[www.persee.fr/web/revues/home/prescript/ article/medi_0751-2708_1998_num_17_ 34_1414].

2010a "Toute littérature est assaut contre la frontière : note sur les embarras historiens d'une rentrée littéraire ", Annales. HSS 65 (2) : 441-467.

2010b Faire profession d'historien.

Paris, Publ. de la Sorbonne.

\section{Boureau, Alain}

2011 En somme. Pour un usage analystique de la scolastique médiévale.

Lagrasse, Verdier.
Brian, Éric

2009 Comment tremble la main invisible.

Incertitude et marché. Paris, Springer.

Brilli, Elisa, Blaise Dufal \& Pierre-Olivier

Dittmar, eds

2010 Ateliers du centre de recherches historiques 6 : Faire l'anthropologie historique du Moyen Âge

[http ://acrh.revues.org/index1911.html].

Buc, Philippe

2003 Dangereux rituels.

De l'histoire médiévale aux sciences sociales.

Paris, Presses universitaires de France.

Burckhardt, Jakob

2008 [1860] Die Cultur der Renaissance

in Italien. Ein Versuch.

München, Beck / Basel, Schwabe.

\section{Burguière, André}

1988 [1978] "L'anthropologie historique", in Jacques Le Goff, ed., La Nouvelle histoire. Paris, Complexe : 137-165.

1995 "L'anthropologie historique ", in François Bédarida, ed., L'Histoire et le métier d'historien en France, 1945-1995. Paris, Éd. de la Maison des sciences de l'homme : 171-186.

Burguière, André et al., eds

1986 Histoire de la famille.

Paris, Armand Colin

Castelli Gattinara, Enrico \& Étienne Anheim

2009 "Jeux d'échelles : une histoire internationale ", Revue de synthèse 4 : 661-678.

Castelli Gattinara, Enrico \& Étienne Anheim, eds

2007 Dimensioni e problemi della ricerca storica 2007 (2): Uso e abuso delle fonti. Roma, Sapienza, Università di Roma.

Cefaï, Daniel, ed.

2010 L'Engagement ethnographique. Paris, Éd. de l'Ehess («En temps \& lieux»16). 
Certeau, Michel de

1975 L'Écriture de l'histoire. Paris,

Gallimard («Bibliothèque des histoires»).

Chastang, Pierre

2008 "L'archéologie du texte médiéval ", Annales. HSS 63 (2) : 245-270.

Colette, Marie-Noëlle

2008 "Guy d'Arezzo et "notre notation musicale moderne": la transmission écrite du chant dans le haut Moyen Âge ",

Revue de Synthèse 129 (3) : 363-387.

\section{Coste, Florent}

2010 "Philippe Descola en Brocéliande», in Elisa Brilli, Blaise Dufal \& Pierre-Olivier Dittmar, eds, Ateliers du centre de recherches historiques 6... [http ://acrh.revues.org/1969].

Dalarun, Jacques

1992 La Sainte et la cité. Micheline de Pesaro (morte en 1356), tertiaire franciscaine.

Rome, École française de Rome.

2007 Vers une résolution de la question franciscaine. La légende ombrienne de Thomas de Celano. Paris, Fayard.

\section{Debaene, Vincent}

2010 L'Adieu au voyage. L'ethnologie française entre science et littérature.

Paris, Gallimard

("Bibliothèque des sciences humaines").

Delacroix, Christian, François Dosse \& Patrick Garcia

2007 Les Courants historiques en France, $X I X^{\top}-X X^{e}$ siècles. Paris, Gallimard

(«Folio. Histoire» 158).

Delacroix, Christian et al., eds

2010 Historiographies. Concepts et débats.

Paris, Gallimard, 2 vol.

(«Folio. Histoire» 179 et 180).

Descola, Philippe

2005 Par-delà nature et culture.

Paris, Gallimard

(«Bibliothèque des sciences humaines »).
Didi-Huberman, Georges

2002 L'Image survivante. Histoire de l'art et temps des fantômes selon Aby Warburg.

Paris, Minuit.

2004 Images malgré tout.

Paris, Minuit.

Dirks, Nicholas B.

2002 «Annals of the Archive :

Ethnographic Notes on the Sources of History ", in Brian Keith Axel, ed., From the Margins. Historical Anthropology and its Future. Durham, Duke University Press : 48-65.

\section{Duby, Georges}

1991 L'Histoire continue. Paris, Odile Jacob.

1996 Féodalité.

Paris, Gallimard («Quarto ).

Dulong, Renaud

1998 Le Témoin oculaire. Les conditions sociales de l'attestation personnelle.

Paris, Éd. de l'EHESS.

2009 "Qu'est-ce qu'un témoin

historique?", Vox-poetica. Théorie et critique

[www.vox-poetica.org/t/articles/dulong.html].

Elias, Norbert

1993 Engagement et distanciation.

Paris, Fayard.

Fabian, Johannes

2006 [1983] Le Temps et les autres.

Comment l'anthropologie construit son objet.

Trad. de l'anglais par Estelle Henry-

Bossonney \& Bernard Müller.

Toulouse, Anacharsis.

Farge, Arlette

1989 Le Goût de l'archive.

Paris, Le Seuil.

Feller, Laurent, Agnès Gramain

\& Florence Weber

2005 La Fortune de Karol.

Marché de la terre et liens personnels

dans les Abruzzes au haut Moyen Âge.

Rome, École française de Rome. 
Ferguson, Wallace K.

2009 [1948] La Renaissance dans la pensée historique. Trad. de l'anglais (États-Unis)

par Jacques Marty. Paris, Payot \& Rivages.

Fritz, Jean-Marie

2000 Paysages sonores du Moyen Âge.

Le versant épistémologique.

Paris, Champion.

Frugoni, Arsenio

1993 [1954] Arnaud de Brescia

dans les sources du XIT siècle.

Trad. de l'italien par Alain Boureau.

Paris, Les Belles Lettres.

Fubini, Riccardo

2001 L'Umanesimo italiano e $i$ suoi storici. Origini rinascimentali - critica moderna.

Milano, Franco Angeli.

\section{Geffray, Christian}

1991 La Cause des armes au Mozambique. Anthropologie d'une guerre civile.

Paris, Karthala.

1997 Le Nom du maître.

Strasbourg, Arcanes.

2001 Trésors. Strasbourg, Arcanes.

\section{Ginzburg, Carlo}

1989 Mythes, emblèmes, traces.

Morphologie et histoire.

Paris, Flammarion.

2001 À distance. Neuf essais sur le point de vue en histoire. Trad. de l'italien par Pierre-Antoine Fabre. Paris, Gallimard ("Bibliothèque des histoires").

2010 Le Fil et les traces. Vrai faux fictif.

Lagresse, Verdier.

Gribaudi, Maurizio, ed.

1998 Espaces, temporalités, stratifications.

Exercices sur les réseaux sociaux.

Paris, Éd. de l'EHESS ("Recherches

d'histoire et de sciences sociales " 84).

Haenel, Yannick

2009 Jan Karski.

Paris, Gallimard («L'infini»).

\section{Halbwachs, Maurice}

1994 [1925] Les Cadres sociaux

de la mémoire.

Paris, Albin Michel.

1997 [1950] La Mémoire collective.

Paris, Albin Michel

2008 [1941] La Topographie légendaire

des Evangiles en Terre Sainte:

essai de mémoire collective.

Paris, Presses universitaires de France.

\section{Hartog, François}

2000 "Le témoin et l'historien",

Gradhiva 27 : 1-14.

2002 Régimes d'historicité.

Présentisme et expériences du temps.

Paris, Le Seuil.

\section{Hollier, Denis}

1991 "La valeur d'usage de l'impossible ", préface à la réédition de la revue Documents.

Paris, Jean-Michel Place.

Huizinga, Johan

2002 [1919] L’Automne du Moyen Âge.

Paris, Payot.

logna-Prat, Dominique

1998 Ordonner et exclure.

Paris, Aubier.

2006 La Maison-Dieu.

Une histoire monumentale de l'Église

au Moyen Âge (800-1200).

Paris, Le Seuil.

Izard, Michel

2003 Moogo. L'émergence d'un espace

étatique ouest-africain au XVI siècle:

étude d'anthropologie historique.

Paris, Karthala.

Jamin, Jean \& Françoise Zonabend, eds

2001-2002 Gradhiva 30-31:

Archives et anthropologie.

Paris, Jean-Michel Place.

\section{Étienne Anheim}


Jolly, Éric

2010 «L'épopée en contexte : variantes et usages politiques de deux récits épiques (Mali/Guinée) ", Annales. HSS 65 (4) : 885-912.

Koselleck, Reinhardt

1990 Le Futur passé. Contribution à la sémantique des temps historiques.

Paris, Éd. de l'EHEss.

1997 L'Expérience de l'histoire.

Trad. de l'allemand par Alexandre Escudier. Paris, Gallimard-Le Seuil

(«Hautes Études»).

\section{LaCapra, Dominick}

1987 History and Criticism.

Ithaca, Cornell University Press.

Laurens, Sylvain \& Frédéric Neyrat

2010 Enquêter: de quel droit?

Menaces sur l'enquête en sciences sociales.

Bellecombe-en-Bauges, Éd. du Croquant.

\section{L'Estoile, Benoît de}

2007 Le Goût des autres.

Paris, Flammarion.

L'Estoile, Benoît de \& Michel Naepels, eds 2004 Critique 680-681 : Frontières de l'anthropologie. Paris, Minuit.

\section{Le Goff, Jacques}

1977 Pour un autre Moyen Âge.

Temps, travail et culture en Occident. Paris, Gallimard

("Bibliothèque des histoires").

1996 Saint Louis. Paris, Gallimard

("Bibliothèque des histoires »).

Le Roy Ladurie, Emmanuel

1975 Montaillou, village occitan, de 1294 à 1324. Paris, Gallimard («Bibliothèque des histoires ").

Leiris, Michel

1996 Miroir de l'Afrique.

Éd. par Jean Jamin.

Paris, Gallimard («Quarto »).
Lett, Didier

2008 Un procès de canonisation

au Moyen Âge. Essai d'histoire sociale.

Paris, Presses universtaires de France.

Lett, Didier, ed.

2008 Médiévales 54 : Frères et sours.

Ethnographie d'un lien de parenté

[http ://medievales.revues.org/4423].

\section{Levi, Giovanni}

1989 Le Pouvoir au village.

Histoire d'un exorciste dans le Piémont

$d u$ XVII siècle. Trad. de l'italien par Monique

Aymard. Paris, Gallimard

("Bibliothèque des histoires").

Loraux, Nicole

2005 La Cité divisée. Paris, Payot,

Magnani, Eliana, ed.

2007 Don et sciences sociales.

Théories et pratiques croisées.

Dijon, Éd. universitaires de Dijon.

Minard, Philippe et al.

2002 « Histoire et anthropologie, nouvelles convergences?", Revue d'histoire moderne et contemporaine 49 (4bis) : 81-120.

Morsel, Joseph

2003 "Les sources sont-elles "le pain de l'historien" ? ", Hypothèses 1 : 271-286.

2007 L'Histoire (du Moyen Âge) est un sport de combat... En collab. avec Christine

Ducourtieux. [Paris], Lamop-Paris 1

[http ://lamop.univ-paris1.fr/IMG/pdf/

SportdecombatMac.pdf].

2009 La Noblesse contre la ville?

Comment faire l'histoire des rapports entre

nobles et citadins en Franconie vers 1500?

Paris, Université Paris 1 Panthéon-

Sorbonne, mémoire d'HDR.

Moulinier, Laurence \& Odile Redon

1998 «Entretien avec Jacques Le Goff:

propos recueillis ", Médiévales 34 : 91-100

[www.persee.fr/web/revues/home/prescript/

article/medi_0751-2708_1998_num_17_

34_1416]. 
Naepels, Michel

1998 Histoires de terres kanakes.

2011 Ethnographie, pragmatique, histoire. Paris, Pub. de la Sorbonne.

Naepels, Michel, ed.,

2010 Annales, Histoire, sciences sociales

65 (4): L'anthropologie face au temps.

Paris, Éd. de l'EHEss.

Nora, Pierre, ed.

1986 Essais d'ego-histoire. Paris, Gallimard

(Bibliothèque des histoires" 68).

Passeron, Jean-Claude

2005 [1991] Le Raisonnement sociologique. Paris, Albin Michel.

Pastoureau, Michel

2010 La Couleur des souvenirs.

Paris, Le Seuil.

Ramage, Maëlle

2009 «Documents, objet et recherche historique : à propos d'un procès de canonisation au Moyen Âge", Revue de synthèse 130 (4) : 697-703.

Revel, Jacques, ed.

1996 Jeux d'échelles.

Paris, Gallimard-Le Seuil

(«Hautes Études»).

Ricœur, Paul

2000 La Mémoire, l'histoire, l'oubli.

Paris, Le Seuil.

Rosaldo, Renato

1986 «From the Door of his Tent:

The Fieldworker and the Inquisitor ", in James Clifford \& George Marcus, eds, Writing Culture. Berkeley, University of California Press : 77-97.

Schmitt, Jean-Claude

2001 Le Corps, les rites, les rêves, le temps. Essais d'anthropologie médiévale. Paris, Gallimard ("Bibliothèque des histoires»).

2002 Le Corps des images.

Essai sur la culture visuelle du Moyen Âge.

Paris, Gallimard ("Le temps des images").

2004 [1979] Le Saint lévrier. Guinefort, guérisseur d'enfants depuis le XIII' siècle. Paris, Flammarion ("Champs» 559). 2010a «L'anthropologie historique de l'Occident médiéval : un parcours ", in Elisa Brilli, Blaise Dufal \& Pierre-Olivier Dittmar, eds, Ateliers du centre de recherches historiques 6... [http ://acrh.revues.org/1926].

2010b L'Invention de l'anniversaire.

Paris, Arkhè.

\section{Stoczkowski, Wiktor}

2007 « Racisme, antiracisme et cosmologie lévi-straussienne: un essai d'anthropologie réflexive ", L'Homme 182 : 7-52.

2008 Anthropologies rédemptrices.

Le monde selon Lévi-Strauss. Paris, Hermann.

Terray, Emmanuel

1995 Une histoire du royaume Abron du Gyaman, des origines à la conquête coloniale. Paris, Karthala.

Thomas, Nicholas

1998 [1989] Hors du temps.

Histoire et évolutionnisme dans le discours anthropologique. Paris, Belin.

Thompson, Edward P.

1963 The Making of the English

Working-Class. London, Victor Gollancz.

Vöss, Jurgen

1972 Das Mittelalter im historischen Denken Frankreichs. Untersuchung zur Geschichte des Mittelaltersbegriffes und der

Mittalterbewertung von der zweiten Hälfte des 16. bis zur Mitte des 19. Jahrhunderts. München, W. Fink.

\section{Weber, Florence}

2008 Manuel de l'ethnographie. Paris, Presses universitaires de France.

Wieviorka, Annette

1998 L'Ère du témoin. Paris, Plon. 
Étienne Anheim, L'historien au pays des merveilles?: histoire et anthropologie au début $d u X X I^{e}$ siècle. - Les liens entre anthropologie et histoire, qui ont été très forts depuis les années 1960, semblent parfois se distendre depuis deux décennies. À partir d'un point de vue localisé, celui d'un historien des derniers siècles du Moyen Âge, cet article cherche à s'interroger sur la réalité actuelle des formes d'échange scientifique entre les deux disciplines. Tout en rappelant l'importance de l'anthropologie historique et des objets de recherche partagés par les historiens et les anthropologues, il cherche à mettre en évidence une autre perspective de travail, en montrant comment les interrogations des anthropologues sur l'historicité, sur la forme et le statut de l'information recueillie durant leur travail de terrain, ou sur leur propre engagement subjectif par rapport à ce terrain, offrent aux historiens, qui sont confrontés à des questionnements parallèles, des ressources nouvelles pour prolonger la fécondité des échanges interdisciplinaires.
Étienne Anheim, Historians in Wonderland? History and Anthropology at the Start of the $2 I^{\text {st }}$ Century. - The links between anthropology and history have been very tight since the 1960 s but seem to have loosened during the past two decades. From a very «localized " viewpoint as a historian of the latter part of the Middle Ages, questions are raised about the current reality of forms of scientific exchanges between these two disciplines. While recalling the importance of historical anthropology and of subjects of research shared by historians and anthropologists, another perspective of work is opened by showing how anthropologists' interrogations about historicity, the form and status of the information gathered during fieldwork, or about their own subjective commitments in relation to the field, provide historians, who face parallel interrogations, with new resources for fertilizing multidisciplinary exchanges. 